\title{
Estudo Comparativo de Modelos de Fissuração Distribuída para Estruturas de Concreto
}

\author{
Comparative Study of Smeared Cracking \\ Models for Concrete Structures
}

\author{
Samuel Silva Penna ${ }^{1}$; Roque L. S. Pitangueira ${ }^{2}$; Jamile Salim Fuina ${ }^{3}$
}

\section{Resumo}

$\mathrm{O}$ artigo mostra como a modelagem constitutiva do concreto evoluiu, desde as tentativas iniciais de caracterizar o meio fissurado como contínuo, passando-se pelos modelos de fissuras distribuídas, de dano e de microplanos, até a tendência atual de conceber diferentes modelos segundo um único arcabouço teórico. Uma formulação genérica para os modelos de fissuração distribuída, que inclui os modelos de direção fixa e rotacional, bem como a degradação em tração e em compressão, é apresentada. Usando esta formulação, três modelos são gerados, especificando as leis de degradação. Um estudo comparativo dos modelos, baseado em simulações computacionais de uma viga submetida a cisalhamento em quatro pontos, é apresentado. Os resultados são comparados, entre si e com resultados experimentais, permitindo uma análise critica dos modelos.

Palavras-chave: Estruturas de Concreto, Método dos Elementos Finitos, Modelos Constitutivos.

\begin{abstract}
The article shows how the constitutive modeling of concrete has evolved since the initial attempts to characterize the medium cracked as continuous, moving from smeared cracking, damage and microplane models, until the current tendency to design different models according to a single theoretical framework. A generic formulation for smeared cracking models, including fixed and rotational models, as well as degradation in tension and in compression, is provided. Using this formulation, three models are generated by specifying the laws of degradation. A comparative study of models, based on computer simulations of a beam subjected to shear at four points, is presented. The results are compared, between themselves and with experimental results, providing a critical analysis of the models.
\end{abstract}

Key words: Concrete Structures, Finite Element Method, Constitutive Models.

\footnotetext{
1 Docente do Depto. de Engenharia de Estruturas da Universidade Federal de Minas Gerais; spenna@dees.ufmg.br

2 Docente do Depto. de Engenharia de Estruturas da Universidade Federal de Minas Gerais; roque@dees.ufmg.br

3 Docente da Faculdade de Engenharia e Arquitetura da Universidade FUMEC; jamile@fumec.br
} 


\section{Introdução}

As características mecânicas e composição física do concreto sólido permitem caracterizá-lo como um material altamente não linear. Tal não linearidade tem origem em fenômenos que tornam o comportamento mecânico do concreto de difícil descrição analítica. A nucleação e propagação de fissuras e o esmagamento do concreto comprimido são alguns dos fenômenos que caracterizam a grande complexidade deste material que tem sido fonte de estudos e pesquisas desde o início do século $\mathrm{XX}$, quando surgiram os primeiros fundamentos teóricos. Inicialmente, o estudo do concreto era baseado em aproximações empíricas e as teorias eram estabelecidas com base em simplificações, que contornavam dificuldades de formulação de uma estrutura teórica mais elaborada. Este cenário se modificou com o desenvolvimento do Método dos Elementos Finitos (MEF). Hipóteses, antes desconsideradas, passaram a ser inseridas em modelos cada vez mais sofisticados com o objetivo de descrever o comportamento do concreto de forma mais realista possível. Assim, muitos e diferentes modelos do MEF foram elaborados ao longo dos anos.

Este artigo apresenta um breve histórico da evolução dos modelos constitutivos para concreto. Esta breve apresentação não pretende mostrar todos os modelos, mas os estudos que mais contribuíram para o avanço nesta área do conhecimento. Uma formulação que generaliza os denominados Modelos de Fissuração Distribuída é então detalhada. A referida formulação é particularizada, gerando três diferentes modelos. Resultados de simulações computacionais com esses modelos são apresentados e comparados, entre si e com resultados experimentais, permitindo uma análise crítica dos mesmos.

\section{Breve Histórico}

Aocorrência, isolada ou combinada, de diferentes fenômenos tornam o comportamento mecânico do concreto de difícil descrição analítica. Tal comportamento é fonte de estudos e pesquisa desde o início do século $\mathrm{XX}$, quando surgiram os primeiros fundamentos teóricos. Inicialmente, o estudo do concreto armado era baseado em aproximações empíricas e as teorias eram estabelecidas com base em simplificações, que contornavam dificuldades de formulação de uma estrutura teórica mais elaborada. Nesse contexto, o método dos elementos finitos surgiu como um recurso geral que ampliou as possibilidades de estudo. Hipóteses, antes simplificadas para serem incorporadas na teoria analítica, passaram a ser inseridas em modelos cada vez mais sofisticados com o objetivo de descrever o comportamento do concreto de forma mais realista possível. Vários aspectos do método dos elementos finitos, quando este é empregado para caracterizar o comportamento não linear do concreto, são estudados desde os primeiros modelos. Um dos primeiros trabalhos nesta área foi o de $\mathrm{NGO}$ e SCORDELIS (1967) que, considerando os materiais como elástico-lineares, incluía representações para as armaduras e fissuras, sendo as trincas inseridas na malha de elementos finitos de forma discreta, assumindo padrões já constatados empiricamente.

O trabalho de NILSON (1968) era semelhante, sendo as trincas inseridas na malha de forma discreta, entretanto, já se considerava o comportamento não linear do material. Até então, nestes modelos iniciais, a idealização de um meio contínuo fissurado, em substituição ao meio contínuo intacto inicial, não havia sido concebida.

A representação do processo de fissuração em modelos planos foi inicialmente tratada por RASHID (1968), que introduzia trincas paralelas distribuídas em regiões internas aos elementos finitos, em contraposição à inserção das fissuras nas interfaces entre elementos. $\mathrm{O}$ material atingia um limite de resistência e, neste ponto, a rigidez da região decaía devido ao início da degradação. Embora limitado, o modelo proposto apresentava facilidade de implementação e já trazia a hipótese fundamental dos modelos de fissuração distribuída: 
a substituição do meio contínuo inicial por um meio, também contínuo, cujas propriedades mecânicas representem uma região com fissuras uniformemente distribuídas.

Modelos subsequentes (SUIDAN e SCHNOBRICH, 1973) mantiveram a rigidez residual transversal como função do módulo de elasticidade transversal inicial, através de um fator de redução denominado fator de retenção ao cisalhamento.

Modelos unidimensionais para tratar o concreto, através de relações momento-curvatura, também já eram usados. No trabalho de MONNIER (1970), por meio de estudos teóricos e experimentais em vigas de concreto, leis não lineares para a relação momento-curvatura foram desenvolvidas. Uma abordagem computacional sobre o assunto foi feita por BLAAUWENDRAAD (1972), que revisou os conceitos adotados até aquele momento e apresentou um modelo unidimensional que considerava efeitos de segunda ordem combinados com relações momento-curvatura.

O uso de parâmetros da mecânica da fratura para modelagem de estruturas de concreto foi iniciado por HILLERBORG et al. (1976). Neste trabalho, o concreto é descrito com um comportamento crescente de tensão até um limite máximo. A partir deste limite, o material entra em regime de abrandamento -- diminuição de tensão acompanhada de aumento de deformação -- que é regido por uma lei não linear baseada na energia de fratura.

No mesmo ano, BAZANT (1976) estudou o abrandamento do concreto e o surgimento de uma banda de fissuração, descrita através da variação da flexibilidade. O monitoramento da flexibilidade também foi estudado por SWARTZ et al. (1978), sendo feita uma análise de sua variação em vigas de concreto submetidas à flexão. A partir de então, os modelos de fissuração distribuída baseados em flexibilidade foram desenvolvidos.

DARWIN e PECKNOLD (1976) apresentaram a modelagem de painéis de concreto armado submetido a cargas cíclicas, usando leis tensãodeformação biaxiais que admitiam degradação tanto em tração como em compressão. Este trabalho foi de grande relevância, pois, até o momento, apenas a degradação do concreto por tração era considerada.

Inspirados em BAZANT (1976), muitos trabalhos (BAZANT e CEODOLIN, 1979, 1980, 1983 ) foram publicados abordando o mesmo tema: o surgimento de uma região fissurada designada como banda de fissuração ou zona de processamento de fissuras. Mas, somente no trabalho de BAZANT e $\mathrm{OH}$ (1983), a teoria foi elaborada admitindo um amolecimento gradual. O modelo considerava a relação constitutiva com amolecimento (como proposto por HILLERBORG et al., 1976) associada a um certo comprimento de banda de fissuração, que era tratado como uma propriedade do material.

A partir destes primeiros modelos, os conceitos de fissuração distribuída se sedimentaram e diversos trabalhos surgiram (DE BORST e NAUTA, 1985; ROTS et al., 1985; e ROTS e DE BORST, 1987), aprimorando as teorias existentes, resultando nos modelos de fissuração fixa e rotacional como conhecidos hoje.

A demanda dos modelos de fissuração distribuída por leis tensão-deformação representativas das regiões degradadas motivou muitos trabalhos, como os de CARREIRA e CHU (1985), CARREIRA e CHU (1986), BOONE et al. (1986) e BOONE e INGRAFFEA (1987), que definem leis tensãodeformação representativas do concreto.

ROTS (1988) apresentou um amplo estudo sobre os modelos de fissuração distribuída, empregando estes modelos para descrever o processo de fraturamento do concreto. No mesmo ano, outro amplo estudo sobre o comportamento não linear do concreto foi apresentado por PROENÇA (1988). Neste trabalho, após descrever modelos elastoplásticos e modelos baseados na mecânica da fratura, Proença apresenta uma formulação variacional para a modelagem constitutiva. 
Em paraleloaos modelos defissuração, a mecânica do dano contínuo (LEMAITRE e DUFAILLY, 1987; LEMAITRE, 1992; LEMAITRE e DESMORAT, 2005) passa a ser usada na modelagem de materiais parcialmente frágeis. O trabalho de LEMAITRE e DUFAILLY (1987) apresenta várias medidas de dano e conclui que a forma mais eficiente de se medir o dano de um material é por meio da quantificação da densidade de defeitos. Entretanto, tal processo é difícil e incerto para os casos em que não se tem uma uniformidade dos defeitos. Além disso, questões relativas ao tamanho e posição da região de observação do dano ainda são muito subjetivas.

Neste cenário, surgem os modelos de dano baseados em deformação equivalente. MAZARS (1984) apresentou um modelo bastante simples, mas eficiente. $\mathrm{O}$ denominado modelo de dano escalar admite que o dano é medido por uma variável escalar, definida como deformação equivalente e calculada com base nas deformações principais positivas.

Na mesma linha de MAZARS (1984), anos mais tarde, outro modelo de dano escalar baseado em deformação equivalente foi proposto por BREKELMANS et al. (1992). O modelo previa que o material poderia apresentar comportamentos distintos em tração e em compressão, o que é essencial para a modelagem do concreto. A proposta associava a tração e a compressão em uma constante usada no cálculo da deformação equivalente.

Os modelos de dano escalar foram a base de muitos trabalhos, podendo ser citados: CHOW e WANG (1987), BAZANT e PIJAUDIER-CABOT (1987), BAZANT e PIJAUDIER-CABOT (1988), DE VREE et al. (1995), GHRIB e TINAWI (1995), PETRANGELI e OZBOLT (1996), LEE et al. (1997), JIRÁSEK e ZIMMERMANN (1998), PITUBA (1998), CAUVIN e TESTA (1999), DE BORST e GUTIERREZ (1999), DE BORST (2002), PITUBA (2003), JUNIOR e VENTURINI (2007), ALVES et al. (2000), SCOTTA et al. (2002),
JIRÁSEK e PATZAK (2002), XIANG et al. (2002) e LEE et al. (2004), além de inúmeros trabalhos e aplicações encontrados na literatura.

Para uma melhor descrição da anisotropia existente no concreto, uma nova proposta, baseada na teoria de microplanos (TAYLOR, 1938), foi apresentada por BAZANT e GAMBAROVA (1984) e BAZANT e OH (1985). Nesta proposta, o ponto material é tratado como um conjunto de microplanos, orientados em diversas direções e posicionados na superfície de uma esfera de raio unitário centrada no referido ponto. As deformações nestes microplanos correspondem à aplicação de uma restrição cinemática e/ou estática ao tensor macroscópico de deformações. Através de relações tensão-deformação, válidas para os microplanos, calculam-se as tensões em cada microplano. Por fim, impondo-se condições de equivalência de energia, obtém-se o estado macroscópico de tensões e uma avaliação da degradação da rigidez.

Uma primeira evolução deste modelo foi proposta por CAROL et al. (1992), que apresentavam a teoria pela primeira vez usando somente restrição cinemática, abandonando assim os modelos de restrição mista. Embora o modelo marcasse uma evolução, apresentava inconsistências que foram corrigidas e apresentadas por OZBOLT et al. (2001). Os modelos de microplanos até então formulados usavam o princípio dos trabalhos virtuais para a equivalência de energia. Mas foi constatado que as leis termodinâmicas não eram plenamente atendidas. Desta forma, CAROL et al. (2001a) e KUHL et al. (2001) apresentaram uma nova estrutura teórica consistente com os princípios termodinâmicos.

Mais recentemente, LEUKART e RAMM (2002, 2006) adotaram uma nova decomposição das deformações macroscópicas, propondo assim uma nova formulação da teoria de microplanos baseada nos princípios da termodinâmica.

O desenvolvimento de modelos seguindo a teoria da plasticidade compreende uma grande parte da modelagem constitutiva de materiais 
em geral. A teoria da plasticidade, amplamente difundida e empregada, constitui um formato teórico consagrado. Para aplicação deste formato, critérios de resistência são adotados de acordo com as características do material que se deseja representar. Assim, além de todos os modelos desenvolvidos especificamente para o concreto, que tentam representar os fenômenos característicos deste material, foram desenvolvidos ainda modelos baseados na teoria clássica da plasticidade. Os fundamentos da teoria da plasticidade para o concreto podem ser visto em obras clássicas como a de CHEN (1982).

Ao longo dos anos, os modelos para o concreto foram se modificando e evoluindo, mas isso não significou que as primeiras formulações caíram em desuso. Nos últimos anos, diversos estudos foram desenvolvidos a partir destes modelos, podendo ser citados: PETRANGELI e OZBOLT (1996), SHAYANFAR et al. (1997), MOSALAM e PAULINO (1997), WEIHE et al. (1998), PITANGUEIRA (1998), LI e ZIMMERMANN (1998), DE BORST (2002), SILVA (2002), SIMÃO (2003), COMI e PEREGO (2004), BORGES e BITTENCOURT (2005), BELNOUE et al. (2007), PALIGA et al. (2007), JUNIOR e VENTURINI (2007), BARAIN e MARI (2007), PI et al. (2007), MATA et al. (2007), DESMORAT et al. (2007a), SRIKANTH et al. (2007), FALEIRO et al. (2008), DUNDAR et al. (2008), PROENÇA e PITUBA (2008), MANZOLI et al. (2008), HE et al. (2008), VOYIADJIS et al. (2008), PAVAN et al. (2009), MENIN et al. (2009), dentre outros.

Cada um destes estudos, com seus respectivos objetivos, formulam o problema de forma diferente com suas particularidades, hipóteses e notações sem uma legítima preocupação em unificar as teorias em um único formato. Entretanto, analisando as principais referências, verifica-se a tendência de unificação dos modelos em uma única estrutura teórica capaz de tratar de forma genérica a modelagem constitutiva. Esta é a principal motivação do trabalho de CAROL et al. (1994) (RIZZI et al.,
1995; CAROL, 1999; CAROL et al., 2001a,b) que, visando criar uma teoria unificada, desenvolveram uma estrutura para modelos constitutivos baseada na degradação elástica da rigidez utilizando os conceitos de superfícies de carregamento e de degradação, e dos seus respectivos gradientes. A estrutura teórica concebida parece contemplar diversos modelos constitutivos.

\section{Modelos de Fissuras Distribuídas}

Os modelos de fissuração distribuída baseiam-se na idealização de um meio contínuo durante todo o processo de análise. Partindo deste meio contínuo, relações entre tensões e deformações são adotadas para acompanhar o processo de fissuração, sendo os limites de resistência do concreto e parâmetros da mecânica da fratura as bases da formulação.

Os modelos tradicionais de fissuração para o concreto tratam o meio material como inicialmente isotrópico e homogêneo e, à medida que o estado de solicitação no material aumenta, seu comportamento é alterado devido ao surgimento de fissuras, que no decorrer da análise se propagam por todo o meio. A partir do início da fissuração, o material passa a ser tratado como ortotrópico e os eixos de ortotropia determinados de acordo com a direção das fissuras.

Os modelos de fissuração distribuída podem ser classificados em duas categorias: modelo de direção de fissuração fixa ou variável. O modelo com direção fixa considera que, uma vez iniciada, a fissura tem orientação fixa durante todo o processo de propagação. Já o modelo com direção variável admite que as fissuras podem rotacionar de acordo com a orientação das deformações principais.

Nos modelos de fissuração distribuída, tradicionalmente, leis tensão deformação são escritas relativamente aos eixos principais de ortotropia $\mathrm{n}, \mathrm{s}, \mathrm{t}$, na forma

$$
\left\{\sigma_{\ell}\right\}=\left[{ }_{\ell}^{s} \mathrm{D}\right]\left\{\varepsilon_{\ell}\right\}
$$


onde $\left\{\sigma_{\ell}\right\}$ é o vetor de tensões no sistema local, $\left[{ }_{\ell}^{s} \mathrm{D}\right]$ é a matriz constitutiva secante local e $\left\{\varepsilon_{\ell}\right\}$ é o vetor de deformações no sistema local. Portanto, para o caso tridimensional, são necessárias nove componentes de rigidez para caracterização do meio fissurado

$$
\left\{\begin{array}{l}
\sigma_{n n} \\
\sigma_{s s} \\
\sigma_{t t} \\
\sigma_{n s} \\
\sigma_{s t} \\
\sigma_{n t}
\end{array}\right\}=\left[\begin{array}{cccccc}
E_{n n} & E_{n s} & E_{n t} & 0 & 0 & 0 \\
E_{n s} & E_{s s} & E_{s t} & 0 & 0 & 0 \\
E_{n t} & E_{s t} & E_{t t} & 0 & 0 & 0 \\
0 & 0 & 0 & G_{n s} & 0 & 0 \\
0 & 0 & 0 & 0 & G_{s t} & 0 \\
0 & 0 & 0 & 0 & 0 & G_{n t}
\end{array}\right]\left\{\begin{array}{c}
\varepsilon_{n n} \\
\varepsilon_{s s} \\
\varepsilon_{t t} \\
\varepsilon_{n s} \\
\varepsilon_{s t} \\
\varepsilon_{n t}
\end{array}\right\}
$$

Na equação 2, $n$ é a direção normal à fissura e $s$ e $t$ são direções tangenciais à fissura, como ilustrado na figura 1.

Figura 1. Sistema de coordenadas da fissura.

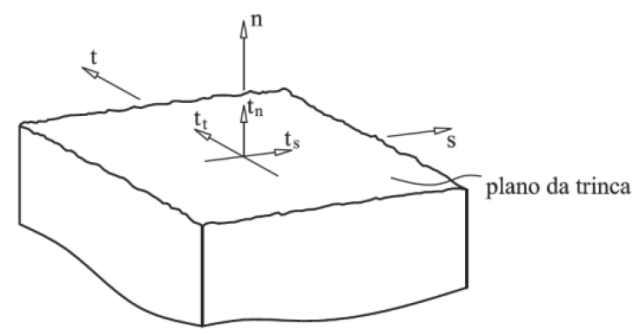

Os modelos iniciais (RASHID, 1968) tratavam as direções normais às fissuras com rigidez nula $\left(E_{n n}\right.$ $=E_{n s}=E_{n t}=G_{n s}=G_{n t}=0$ ). Desta forma, as tensões associadas a estas componentes de rigidez $\left(\sigma_{n n}, \sigma_{n s}\right.$, $\left.\sigma_{n t}\right)$, no momento da abertura da trinca, decaíam de um valor limite para zero. Logo, para o caso bidimensional, tinha-se

$$
\left\{\sigma_{\ell}\right\}=\left\{\begin{array}{l}
\sigma_{n n} \\
\sigma_{s s} \\
\sigma_{n s}
\end{array}\right\} ;\left\{\varepsilon_{\ell}\right\}=\left\{\begin{array}{l}
\varepsilon_{n n} \\
\varepsilon_{s s} \\
\gamma_{n s}
\end{array}\right\} ;\left[{ }_{\ell}^{s} D\right]=\left[\begin{array}{lll}
0 & 0 & 0 \\
0 & E & 0 \\
0 & 0 & 0
\end{array}\right] \quad(3 \mathrm{a}, \mathrm{b}, \mathrm{c})
$$

Estas primeiras aproximações não exprimiam a realidade observada, de que mesmo depois de fissurada, uma peça estrutural ainda era capaz de transmitir esforços normais e tangenciais. A transformação do meio material, inicialmente linearelástico e isotrópico, em um meio ortotrópico com rigidez nula implicava em fortes descontinuidades, que acarretavam em instabilidades numéricas dos modelos. Modelos subsequentes (SUIDAN e SCHNOBRICH, 1973) mantiveram a rigidez residual transversal $G_{n s}$ e $G_{n t}$ como função do módulo de elasticidade transversal inicial $G$, através de um fator de redução denominado fator de retenção ao cisalhamento, designado por $\beta_{\mathrm{r}}$. Portanto, para o caso plano, tem-se

$$
\left[{ }_{f}^{S} D\right]=\left[\begin{array}{ccc}
0 & 0 & 0 \\
0 & E & 0 \\
0 & 0 & \beta_{\mathrm{r} G} \mathrm{G}
\end{array}\right]
$$

$\operatorname{com} \beta_{\mathrm{r}}$ variando de zero a um.

Analogamente, uma evolução do modelo considerava também um fator de redução do módulo de elasticidade nas direções normais à fissura, alterando 4 para

$$
\left[{ }_{\ell}^{s} D\right]=\left[\begin{array}{ccc}
\alpha_{\mathrm{r}} E & 0 & 0 \\
0 & E & 0 \\
0 & 0 & \beta_{\mathrm{r}} \mathrm{G}
\end{array}\right]
$$

$\operatorname{com} \alpha_{\mathrm{r}}$ sendo um fator de redução variando de zero a um.

BAZANT e OH (1983), usando as mesmas ideias, mantiveram a redução do módulo de rigidez normal à fissura $\left(E_{n n}\right)$, além de introduzirem também os módulos de rigidez fora da diagonal da matriz constitutiva, considerando assim o efeito de Poisson após a abertura da trinca. A formulação baseia-se na inversão de uma relação de flexibilidade e considera somente degradação por tração.

O modelo de HILLERBORG et al. (1976) também considerava somente a degradação por tração e a propagação de fissuras era estudada em função das tensões principais, que iam do regime elástico até a ruptura.

Baseando-se no trabalho de BAZANT e $\mathrm{OH}$ (1983), ROTS et al. (1985) desenvolveram um modelo que considerava, não somente a degradação na direção normal à fissura, mas também na direção tangente ao plano de fissuração, de tal modo que a relação tensão-deformação na direção das fissuras era dada por:

$$
\left[{ }_{\ell}^{S} D\right]=\frac{1}{\left(1-v^{2} \alpha_{\mathrm{r}}\right)}\left[\begin{array}{ccc}
\alpha_{\mathrm{r}} E & v \alpha_{\mathrm{r}} E & 0 \\
v \alpha_{\mathrm{r}} E & E & 0 \\
0 & 0 & \frac{\left(1-v^{2} \alpha_{\mathrm{r}}\right) \beta_{\mathrm{r}} G}{2(1+v)}
\end{array}\right]
$$


A ideia da inversão da flexibilidade já estava consolidada e inúmeros trabalhos desde então foram desenvolvidos (ROTS et al., 1985; ROTS e DE BORST, 1987; BAZANT e PIJAUDIERCABOT, 1988; BAZANT e LIN, 1988; ROTS e BLAAUWENDRAAD, 1989; PETRANGELI e OZBOLT, 1996; dentre outros).

A seguir, será descrita uma generalização dos modelos de fissuração distribuída baseados em inversão de flexibilidade, apresentada no trabalho de PITANGUEIRA (1998).

Partindo da relação entre tensões $\left(\left\{\sigma_{\ell}\right\}\right)$ e deformações $\left(\left\{\varepsilon_{\ell}\right\}\right)$ em um sistema de coordenadas local, orientado na direção de fissuração (Figura 2), em termos da flexibilidade $\left(\left[{ }_{\ell}^{s} C\right]\right)$, dada por

$$
\left\{\varepsilon_{\ell}\right\}=\left[{ }_{\ell}^{S} C\right]\left\{\sigma_{\ell}\right\}
$$

pode-se desenvolver as equações que permitem obter o tensor constitutivo secante local.

Figura 2. Eixos locais e globais

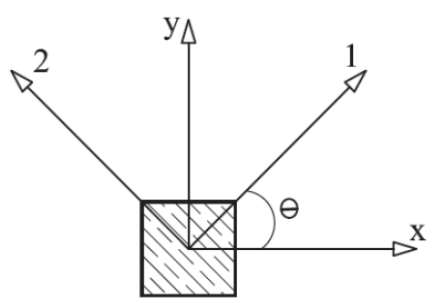

Admitindo que o material seja ortotrópico nas direções principais de deformação, para o caso bidimensional de estado plano de tensão, tem-se

$$
\left[{ }_{\ell}^{S} C\right]=\left[\begin{array}{ccc}
\frac{1}{E_{1}} & -\frac{v_{12}}{E_{2}} & 0 \\
-\frac{v_{21}}{E_{1}} & \frac{1}{E_{2}} & 0 \\
0 & 0 & \frac{1}{G_{12}}
\end{array}\right]
$$

Invertendo $\left[{ }_{\ell}^{S} C\right]$, tem-se o tensor constitutivo secante local dado por

$$
\left[{ }_{\ell}^{S} D\right]=\frac{1}{1-v_{12} v_{21}}\left[\begin{array}{ccc}
E_{1} & v_{12} E_{1} & 0 \\
v_{21} E_{2} & E_{2} & 0 \\
0 & 0 & \left(1-v_{12} v_{21}\right) G_{12}
\end{array}\right] \text { (9) }
$$

Supondo que os módulos de elasticidade nas direções locais são obtidos de forma independente e de acordo com o estado de deformação corrente, o modelo obtido permite considerar a degradação tanto em tração quanto em compressão. Entretanto, verifica-se que a matriz, dada em 9 é assimétrica e, portanto, um processo de simetrização, como sugerido por BAZANT e OH (1983), é adotado.

A matriz de flexibilidade simétrica pode ser obtida tomando as componentes do acoplamento, devido ao efeito de Poisson, como um único valor. Este passa a ser uma função do módulo de elasticidade inicial $\left(E_{0}\right)$ e de um coeficiente de Poisson igual para todas as direções do material $(v)$. Logo, em estado plano de tensão, adota-se

$$
\frac{v_{21}}{E_{1}}=\frac{v_{12}}{E_{2}}=\frac{v}{E_{0}}
$$

resultando em uma matriz de flexibilidade igual a

$$
\left[{ }_{\ell}^{s} C\right]=\left[\begin{array}{ccc}
\frac{1}{E_{1}} & -\frac{v}{E_{0}} & 0 \\
-\frac{v}{E_{0}} & \frac{1}{E_{2}} & 0 \\
0 & 0 & \frac{1}{G_{12}}
\end{array}\right]
$$

Invertendo 11 tem-se a matriz constitutiva secante local dada por

$$
\left[{ }_{f}^{s} D\right]=\frac{1}{1-\frac{E_{1} E_{2}}{E_{0}^{2}} v^{2}}\left[\begin{array}{ccc}
E_{1} & \frac{v E_{1} E_{2}}{E_{0}} & 0 \\
\frac{v E_{1} E_{2}}{E_{0}} & E_{2} & 0 \\
0 & 0 & \left(1-\frac{E_{1} E_{2}}{E_{0}^{2}} v^{2}\right) G_{12}
\end{array}\right]
$$

O módulo de elasticidade transversal, segundo a hipótese da simetrização, fica

$$
G_{12}=\frac{E_{0} E_{1} E_{2}}{E_{0} E_{1}+E_{0} E_{2}+2 v E_{1} E_{2}} \geq \beta_{r} \frac{E_{0}}{2(1+v)}
$$

A limitação do valor mínimo do módulo de elasticidade transversal, para considerar a rugosidade entre as faces da fissura, pode ser feita em função do módulo original, por 


$$
G_{12} \geq \beta_{r} G_{0}
$$

onde

$$
\mathrm{G}_{0}=\frac{\mathrm{E}_{0}}{2(1+v)}
$$

e $\beta_{\mathrm{r}}$ é um valor entre zero e um.

As matrizes de flexibilidade e as matrizes constitutivas secantes dadas acima foram obtidas para o sistema local que, como já admitido, está alinhado com as direções das deformações principais. Entretanto, os tensores constitutivos também devem ser obtidos no sistema global.

Para transformar tensões entre os sistemas (local e global), ilustrados na figura 2, usa-se

$$
\left\{\sigma_{\ell}\right\}=\left[\mathrm{T}_{\sigma}\right]\left\{\sigma_{g}\right\}
$$

onde $\left\{\sigma_{q}\right\}$ são as tensões no sistema global e $\left[\mathrm{T}_{\sigma}\right]$ é a matriz de transformação de tensões. Invertendo e transpondo $\left[\mathrm{T}_{\sigma}\right]$, obtém-se a matriz que permite transformar deformações entre sistemas, usando

$$
\left[\mathrm{T}_{\varepsilon}\right]=\left[\mathrm{T}_{\sigma}\right]^{-\mathrm{T}}
$$

onde

$$
\left\{\varepsilon_{\varepsilon}\right\}=\left[\mathrm{T}_{\varepsilon}\right]\left\{\varepsilon_{g}\right\} .
$$

Substituindo as matrizes de transformação na relação entre tensões e deformações locais, tem-se

$$
\left[\mathrm{T}_{\sigma}\right]\left\{\sigma_{g}\right\}=\left[{ }_{\ell}^{s} \mathrm{D}\right]\left[\mathrm{T}_{\varepsilon}\right]\left\{\varepsilon_{g}\right\}
$$

Logo, tem-se

$$
\left\{\sigma_{\mathcal{g}}\right\}=\left[\mathrm{T}_{\varepsilon}\right]^{\mathrm{T}}\left[{ }_{\ell}^{s} \mathrm{D}\right]\left[\mathrm{T}_{\varepsilon}\right]\left\{\varepsilon_{\mathcal{g}}\right\} .
$$

Reescrevendo 20, tem-se

$$
\left\{\sigma_{g}\right\}=\left[{ }_{g}^{s} D\right]\left\{\varepsilon_{g}\right\} .
$$

Sendo que

$$
\left[{ }_{g}^{s} \mathrm{D}\right]=\left[\mathrm{T}_{\varepsilon}\right]^{\mathrm{T}}\left[{ }_{\varepsilon}^{s} \mathrm{D}\right]\left[\mathrm{T}_{\varepsilon}\right] .
$$

é a matriz constitutiva secante global.

A figura 3 apresenta a sequência do processo de transformações de deformações e tensões ao longo da análise.
Em um processo incremental-iterativo de análise não linear baseado em formulação secante, as equações acima são suficientes. Entretanto, para o caso da formulação tangente desse processo, que requer o cálculo de rigidez tangente incremental, uma relação diferencial entre tensões e deformações deve ser obtida. Assim sendo, partindo da relação de transformação de tensões (equação 16), tem-se

$$
\left\{\sigma_{g}\right\}=\left[\mathrm{T}_{\sigma}\right]^{-1}\left\{\sigma_{\ell}\right\}
$$

Figura 3. Transformações de deformações e tensões.

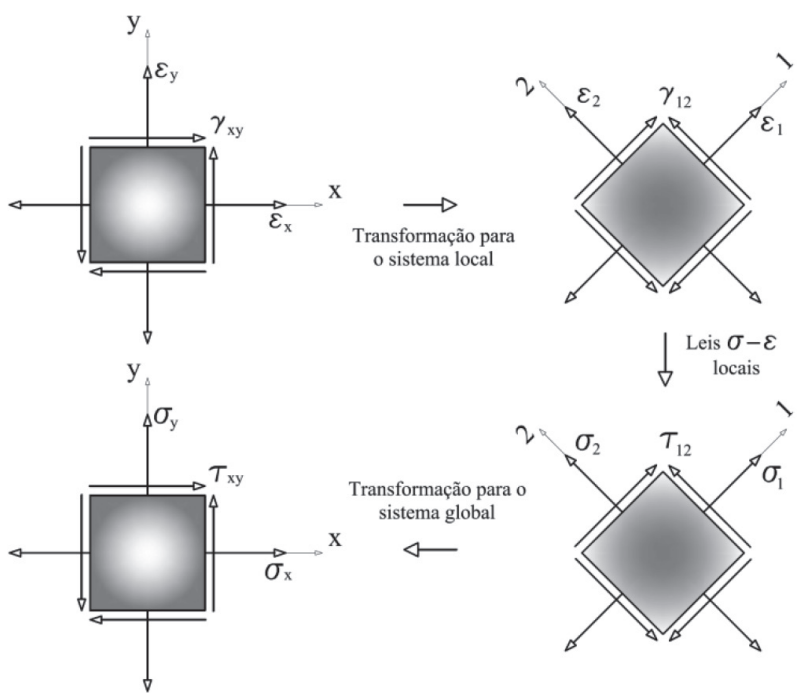

substituindo a relação 17 na equação 25 , tem-se

$$
\left\{\sigma_{g}\right\}=\left[\mathrm{T}_{\varepsilon}\right]^{\mathrm{T}}\left\{\sigma_{\ell}\right\}
$$

Diferenciando 26 em relação a $\left\{\varepsilon_{g}\right\}$, tem-se

$$
\mathrm{d}\left\{\sigma_{g}\right\}=\frac{\partial}{\partial\left\{\varepsilon_{g}\right\}}\left(\left[\mathrm{T}_{\varepsilon}\right]^{\mathrm{T}}\left\{\sigma_{\ell}\right\}\right) \mathrm{d}\left\{\varepsilon_{g}\right\}
$$

ou

$$
\mathrm{d}\left\{\sigma_{g}\right\}=\left[\left[\mathrm{T}_{\varepsilon}\right]^{\mathrm{T}} \frac{\partial\left\{\sigma_{\ell}\right\}}{\partial\left\{\varepsilon_{q}\right\}}+\frac{\partial\left[\mathrm{T}_{\varepsilon}\right]^{\mathrm{T}}}{\partial\left\{\varepsilon_{g}\right\}}\left\{\sigma_{\ell}\right\}\right] \mathrm{d}\left\{\varepsilon_{g}\right\},
$$

que pode ser escrito como

$$
\mathrm{d}\left\{\sigma_{g}\right\}=\left[\left[\mathrm{T}_{\varepsilon}\right]^{\mathrm{T}} \frac{\partial\left\{\sigma_{\ell}\right\}}{\partial\left\{\varepsilon_{\ell}\right\}}\left[\mathrm{T}_{\varepsilon}\right]+\frac{\partial\left[\mathrm{T}_{\varepsilon}\right]^{\mathrm{T}}}{\partial\left\{\varepsilon_{g}\right\}}\left\{\sigma_{\ell}\right\}\right] \mathrm{d}\left\{\varepsilon_{g}\right\},
$$

ou 


$$
\mathrm{d}\left\{\sigma_{g}\right\}=\left[\left[\mathrm{T}_{\varepsilon}\right]^{\mathrm{T}}\left[{ }_{\ell}^{\mathrm{t}} \mathrm{D}\right]\left[\mathrm{T}_{\varepsilon}\right]+\frac{\partial\left[\mathrm{T}_{\varepsilon}\right]^{\mathrm{T}}}{\partial\left\{\varepsilon_{g}\right\}}\left\{\sigma_{\ell}\right\}\right] \mathrm{d}\left\{\varepsilon_{g}\right\},
$$

em que

$$
\left[{ }_{\ell}^{\mathrm{t}} \mathrm{D}\right]=\frac{\partial\left\{\sigma_{\ell}\right\}}{\partial\left\{\varepsilon_{\ell}\right\}}
$$

é a matriz constitutiva tangente no sistema local de coordenadas, e a parcela $\frac{\partial\left[\mathrm{T}_{\varepsilon}\right]^{\mathrm{T}}}{\partial\left\{\varepsilon_{q}\right\}}\left\{\sigma_{\ell}\right\}$ representa a influência de possíveis mudanças na matriz de transformação durante o processo incrementaliterativo.

Para melhor entendimento do tensor constitutivo tangente local, toma-se o diferencial da relação tensão-deformação local, dado por

$$
\mathrm{d}\left\{\sigma_{\ell}\right\}=\frac{\partial\left\{\sigma_{\ell}\right\}}{\partial\left\{\varepsilon_{\ell}\right\}} \mathrm{d}\left\{\varepsilon_{\ell}\right\},
$$

sendo $\frac{\partial\left\{\sigma_{\ell}\right\}}{\partial\left\{\varepsilon_{\ell}\right\}}$ obtido pela derivação da equação $1, \log o$

$$
\frac{\partial\left\{\sigma_{\ell}\right\}}{\partial\left\{\varepsilon_{\ell}\right\}}=\frac{\partial}{\partial\left\{\varepsilon_{\ell}\right\}}\left(\left[{ }_{\ell}^{S} \mathrm{D}\right]\left\{\varepsilon_{\ell}\right\}\right)=\left[{ }_{\ell}^{S} \mathrm{D}\right]+\frac{\partial\left[{ }_{\ell}^{S} \mathrm{D}\right]}{\partial\left\{\varepsilon_{\ell}\right\}}\left\{\varepsilon_{\ell}\right\},
$$

onde $\left[{ }_{\ell}^{\mathrm{s}} \mathrm{D}\right]$ é a matriz constitutiva secante local simétrica.

Entretanto, a parcela $\frac{\partial\left[\left[_{\ell}^{s} \mathrm{D}\right]\right.}{\partial\left\{\varepsilon_{\ell}\right\}}\left\{\varepsilon_{\ell}\right\}$ é não simétrica sendo, para o caso bidimensional, dada por

$$
\frac{\partial\left[_{\ell}^{s} \mathrm{D}\right]}{\partial\left\{\varepsilon_{\ell}\right\}}\left\{\varepsilon_{\ell}\right\}=\left[\begin{array}{ccc}
\frac{\partial_{\ell}^{s} \mathrm{D}_{11}}{\partial \varepsilon_{1}} \varepsilon_{1}+\frac{\partial_{\ell}^{s} \mathrm{D}_{12}}{\partial \varepsilon_{1}} \varepsilon_{2} & \frac{\partial_{\ell}^{s} \mathrm{D}_{11}}{\partial \varepsilon_{2}} \varepsilon_{1}+\frac{\partial_{\ell}^{s} \mathrm{D}_{12}}{\partial \varepsilon_{2}} \varepsilon_{2} & 0 \\
\frac{\partial_{\ell}^{s} \mathrm{D}_{12}}{\partial \varepsilon_{1}} \varepsilon_{1}+\frac{\partial_{\ell}^{s} \mathrm{D}_{22}}{\partial \varepsilon_{1}} \varepsilon_{2} & \frac{\partial_{\ell}^{s} \mathrm{D}_{12}}{\partial \varepsilon_{2}} \varepsilon_{1}+\frac{\partial_{\ell}^{s} \mathrm{D}_{22}}{\partial \varepsilon_{2}} \varepsilon_{2} & 0 \\
0 & 0 & 0
\end{array}\right] \text { (34) }
$$

Na equação 30, o termo dependente da variação da direção do sistema local, no caso bidimensional, varia de acordo com um ângulo $\Theta$ (Figura 2), logo, tem-se

$$
\frac{\partial\left[\mathrm{T}_{\varepsilon}\right]^{\mathrm{T}}}{\partial\left\{\varepsilon_{q}\right\}}\left\{\sigma_{\ell}\right\}=\frac{\partial\left[\mathrm{T}_{\varepsilon}\right]^{\mathrm{T}}}{\partial \theta}\left\{\sigma_{\ell}\right\}\left(\frac{\partial \theta}{\partial \varepsilon_{q}}\right)^{\mathrm{T}},
$$

que é simétrico se houver coaxialidade entre tensões e deformações principais ao longo do processo.

A equação 30 pode agora ser reescrita em termos da equação 35 , portanto, tem-se

$$
\mathrm{d}\left\{\sigma_{\mathcal{g}}\right\}=\left[\left[\mathrm{T}_{\varepsilon}\right]^{\mathrm{T}}\left[{ }_{\ell}^{\mathrm{t}} \mathrm{D}\right]\left[\mathrm{T}_{\varepsilon}\right]+\frac{\partial\left[\mathrm{T}_{\varepsilon}\right]^{\mathrm{T}}}{\partial \theta}\left\{\sigma_{\ell}\right\}\left(\frac{\partial \theta}{\partial \varepsilon_{g}}\right)^{\mathrm{T}}\right] \mathrm{d}\left\{\varepsilon_{g}\right\}
$$

Para o caso do modelo com direção de fissuração fixa, a parcela dependente da variação da direção, na equação 36 , se anula, uma vez que a direção de propagação, no instante do surgimento da fissura, é tomada como constante até o fim da análise. Portanto, neste caso, a relação constitutiva tangente global é dada por

$$
\mathrm{d}\left\{\sigma_{g}\right\}=\left[\mathrm{T}_{\varepsilon}\right]^{\mathrm{T}}\left[{ }_{\ell}^{\mathrm{t}} \mathrm{D}\right]\left[\mathrm{T}_{\varepsilon}\right] \mathrm{d}\left\{\varepsilon_{g}\right\} .
$$

Os modelos rotacionais admitem que a direção principal de deformação continua mudando após o surgimento das primeiras trincas. Desta forma, a parcela dependente da variação da direção, dada na equação 35 , deve ser contabilizada. Para o caso bidimensional, impondo condição de coaxialidade entre deformações $\left(\varepsilon_{1}\right.$ e $\left.\varepsilon_{2}\right)$ e tensões principais $\left(\sigma_{1}\right.$ e $\left.\sigma_{2}\right)$, tem-se

$$
\frac{\partial\left[T_{\varepsilon}\right]^{\mathrm{T}}}{\partial \theta}\left\{\sigma_{\ell}\right\}\left(\frac{\partial \theta}{\partial \varepsilon_{q}}\right)^{\mathrm{T}}=\frac{1}{2} \frac{\gamma_{x y}\left(\sigma_{1} \sigma_{2}\right) \operatorname{sen} 2 \theta}{2\left(\varepsilon_{x} \varepsilon_{y}\right)+\gamma_{\mathrm{xy}}^{2}}\left[\begin{array}{ccc}
1 & -1 & -\operatorname{ctg} 2 \theta \\
-1 & 1 & \operatorname{ctg} 2 \theta \\
-\operatorname{ctg} 2 \theta & \operatorname{ctg} 2 \theta & \operatorname{ctg}^{2} 2 \theta
\end{array}\right] .
$$

$\mathrm{Na}$ generalização acima discutida, o acompanhamento do processo de degradação depende dos valores dos módulos de elasticidade secantes $\left(E_{\mathrm{i}}^{\mathrm{s}}\right)$ e tangentes $\left(E_{\mathrm{i}}^{\mathrm{t}}\right)$ nas direções $\mathrm{i}$ de ortotropia. Estes valores dependem do estado corrente de deformações $\left(E_{i}^{s}=E_{i}^{s}\left(\varepsilon_{i}\right), E_{i}^{t}=E_{i}^{t}\left(\varepsilon_{i}\right)\right)$ e, portanto, podem ser obtidos a partir de leis tensãodeformação escalares, como ilustra a figura 4.

Figura 4. Lei tensão-deformação.

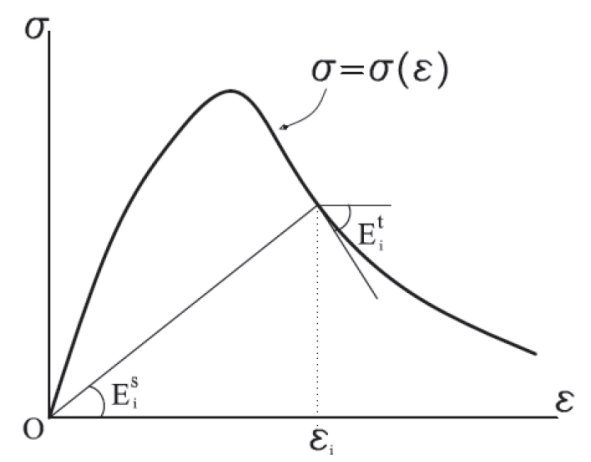


Estudando os sinais das deformações nas direções de ortotropia, pode-se investigar a degradação tanto em tração quanto em compressão, sendo necessárias somente leis específicas para regiões de tração e de compressão.

O modelo de fissuração distribuída escolhido para o estudo comparativo a seguir apresentado assume as seguintes hipóteses: (1) o sistema local de observação coincide com as direções principais de deformações; (2) o acoplamento devido ao efeito de Poisson é descrito por um único coeficiente, válido para todas as direções; (3) os módulos de elasticidade transversais são dados em função dos longitudinais e do coeficiente de Poisson único, mas nunca se tornam inferiores a $0,001 \mathrm{G}_{0}$, onde $\mathrm{G}_{0}$ é o módulo de elasticidade transversal inicial.

Como dito acima, o modelo permite diferentes hipóteses para as leis tesão-deformação locais, responsáveis pela história de degradação dos módulos de elasticidade longitudinais. Três combinações de leis tesão-deformação para regiões de tração e de compressão são aqui usadas, conforme mostra a Figura 5.

\section{Modelo Carreira-Ingraffea}

Neste caso, combinam-se as leis propostas por CARREIRA e CHU (1985), para compressão, e por BOONE e INGRAFFEA (1987), para tração, conforme ilustra a Figura 5(a). Como destacado na figura, os parâmetros necessários à descrição das relações tensão-deformação são: módulo de elasticidade inicial $\left(\mathrm{E}_{0}\right)$; tensão limite à compressão $\left(f_{c}\right)$; tensão limite à tração $\left(f_{t}\right)$ e energia de fratura por unidade de área de fissura $\left(\mathrm{g}_{\mathrm{f}}\right)$, considerada como $\mathrm{g}_{\mathrm{f}}$ $=\mathrm{G}_{\mathrm{f}} \mathrm{h}$, onde $\mathrm{h}$ é o comprimento característico do material e $G_{f}$ a energia de fratura por unidade de comprimento de fissura.

\section{Modelo Carreira-Carreira}

Este modelo combina a lei proposta por CARREIRA e CHU (1985) para compressão com a proposta por CARREIRA e CHU (1986), para tração. Neste caso, conforme mostra a Figura 5(b), os parâmetros necessários para descrever as leis tensão-deformação são: módulo de elasticidade inicial $\left(\mathrm{E}_{0}\right)$; tensão limite à compressão $\left(\mathrm{f}_{\mathrm{c}}\right)$; tensão limite à tração $\left(\mathrm{f}_{\mathrm{t}}\right)$; deformação correspondente ao limite de compressão $\left(\varepsilon_{c}\right)$ e deformação correspondente ao limite de tração $\left(\varepsilon_{t}\right)$. Os ramos descendentes das leis são descritos pelas relações de proximidade entre $\varepsilon_{c}$ e $\varepsilon_{t}$ e seus correspondentes elástico-lineares: $\mathrm{f}_{\mathrm{c}} / \mathrm{E}_{0}$ e $\mathrm{f}_{\mathrm{t}} / \mathrm{E}_{0}$, respectivamente.

\section{Modelo Bilinear}

Neste caso, as leis bilineares mostradas na Figura 5(c) são adotadas. Para descrição de tais leis, os seguintes parâmetros são usados: módulo de elasticidade inicial $\left(\mathrm{E}_{0}\right)$; tensão limite à compressão $\left(\mathrm{f}_{\mathrm{c}}\right.$; módulo de elasticidade do ramo descendente para compressão $\left(E_{2}\right)$; tensão limite à tração $\left(f_{t}\right)$ e energia de fratura por unidade de área de fissura $\left(\mathrm{g}_{\mathrm{f}}\right)$, novamente considerada como $\mathrm{g}_{\mathrm{f}}=\mathrm{G}_{\mathrm{f}} / \mathrm{h}$, onde h é o comprimento característico do material e $\mathrm{G}_{\mathrm{f}}$ a energia de fratura por unidade de comprimento de fissura.

Os modelos constitutivos acima descritos foram implementados no INSANE (Interactive Structural Analysis Environment), um programa de elementos finitos de código aberto (PITANGUEIRA, 2008), que possui todos os recursos necessários à análise fisicamente não-linear aqui apresentada. 
Figura 5. Leis Tensão-Deformação adotadas nos Modelos

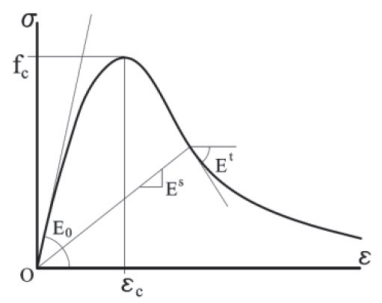

(a) Modelo Carreira-Ingraffea

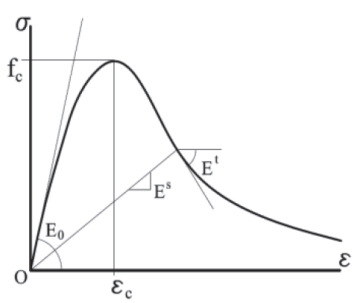

(b) Modelo Carreira- Carreira
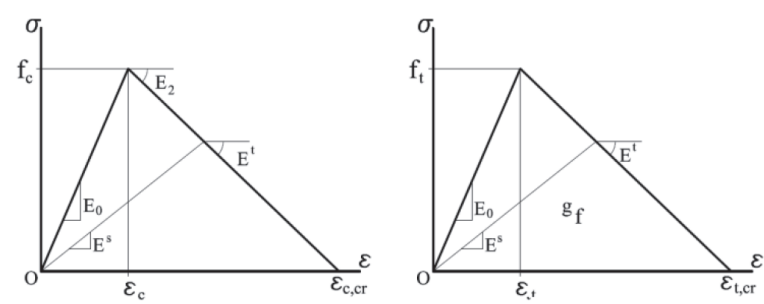

(c) Modelo Bilinear

\section{Estudo Comparativo}

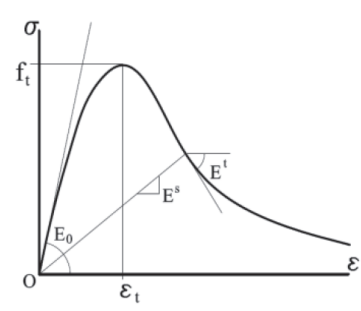

Analisa-se aqui a viga sob cisalhamento em quatro pontos mostrada na Figura 6. Esta viga foi estudada por SCHLANGEN (1993). Neste estudo experimental, observou-se a amplificação dos deslocamentos relativos horizontais (CMOD) e vertical (CMSD) dos pontos externos da trinca inicial, conforme mostra detalhe da Figura.
Figura 6. Viga sob cisalhamento em quatro pontos
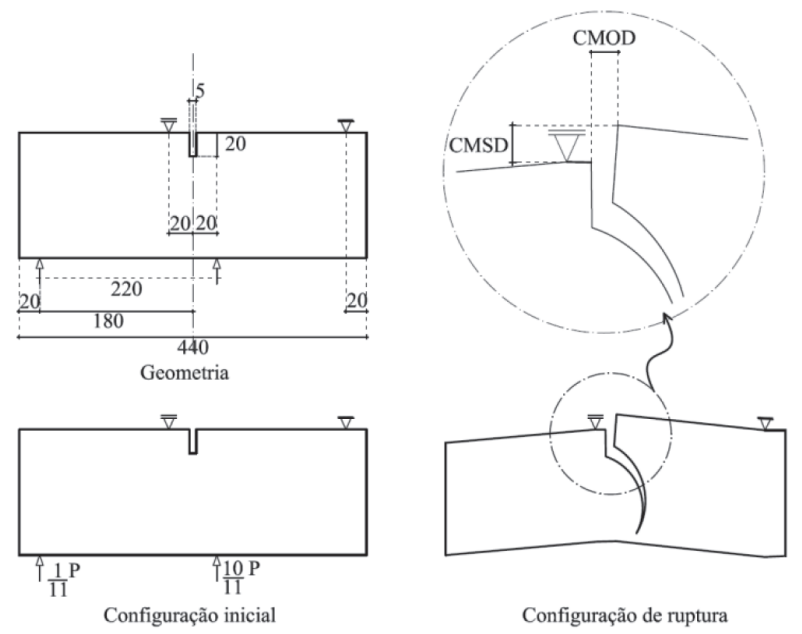

Para a análise numérica aqui apresentada, utilizou-se elementos finitos quadrilaterias de 4 nós de estado plano de tensões, com a discretização mostrada na Figura 7. Consoante com os dados experimentais de SCHLANGEN (1993), foram adotados os seguintes parâmetros para os três modelos constitutivos acima descritos (ver Figura 5): $E_{0}=30000 \mathrm{~N} / \mathrm{mm}^{2} ; f_{c}=46,6 \mathrm{~N} / \mathrm{mm}^{2}$; $f_{t}=3,44 \mathrm{~N} / \mathrm{mm}^{2} ; G_{f}=0,135 \mathrm{~N} / \mathrm{mm} ; \mathrm{h}=12 \mathrm{~mm} ;$ $\varepsilon_{c}=0,003 ; \varepsilon_{t}=0,00028 ; E_{2}=3000 \mathrm{~N} / \mathrm{mm}^{2} ; v=0,15$.

Figura 7. Malha de Elementos Finitos para a Viga da Figura 6

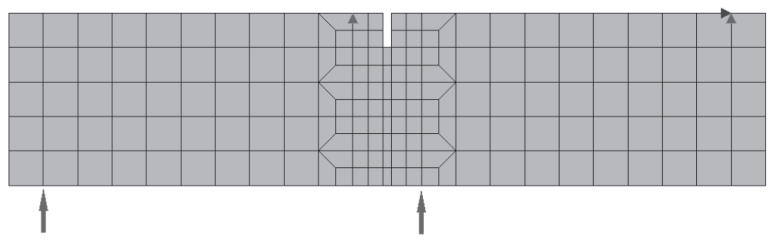

A Figura 8 mostra os resultados da análise numérica para os três modelos, considerando as hipóteses de direção de fissuração fixa e rotacional. Tais resultados foram obtidos com o método incremental-iterativo de deslocamento generalizado proposto por YANG e SHIEH (1990), utilizando-se carga de referência $P=40 \mathrm{kN}$ e tolerância para erro em cada passo de 0.0001 . A figura também mostra 
o espectro de resultados experimentais obtido por SCHLANGEN (1993).

A observação dos resultados da Figura 8 permite constatar:

(1) excelente concordância dos modelos numéricos com os resultados experimentais, para o regime préfissuração;

(2) boas estimativas da carga limite obtidas com os Modelos Carreira-Ingraffea e Carreira-Carreira;

(3) que, para o modo de abertura, caracterizado pelos valores de CMOD (Figura 8(a)), os Modelos Carreira-Ingraffea e Carreira-Carreira, com direção de fissuração fixa, descreveram bem o regime de abrandamento até aproximadamente metade da carga máxima, quando o Modelo Carreira-Carreira revelou-se mais dúctil, desviando-se dos resultados experimentais, e o Modelo Carreira-Ingraffea tornou-se mais frágil, mas ainda se mantendo dentro da faixa de valores experimentais;

(4) que, para o modo de abertura, o Modelo Carreira-Ingraffea, com direção de fissuração rotacional, também descreveu adequadamente o regime de abrandamento durante todo o processo de propagação;

(5) que, para o modo de deslizamento no plano, caracterizado pelos valores de CMSD (Figura 9(b)), todos os modelos apresentaram comportamento pós-fissuração mais frágil que o observado experimentalmente;

(6) que os Modelos Bilineares não foram capazes de estimar adequadamente nem a carga limite nem o comportamento pós-fissuração;

(7) que, para as três leis tensão-deformação adotadas, os modelos de direção rotacional resultam em estimativas de carga superiores àquelas obtidas com os modelos de direção fixa;

(8) que, para as três leis tensão-deformação, os modelos de direção rotacional apresentaram comportamentos pós-fissuração mais dúcteis que os obtidos com os modelos de direção fixa.
Figura 8: Resultados Numéricos e Experimentais para a Viga da Figura 6.

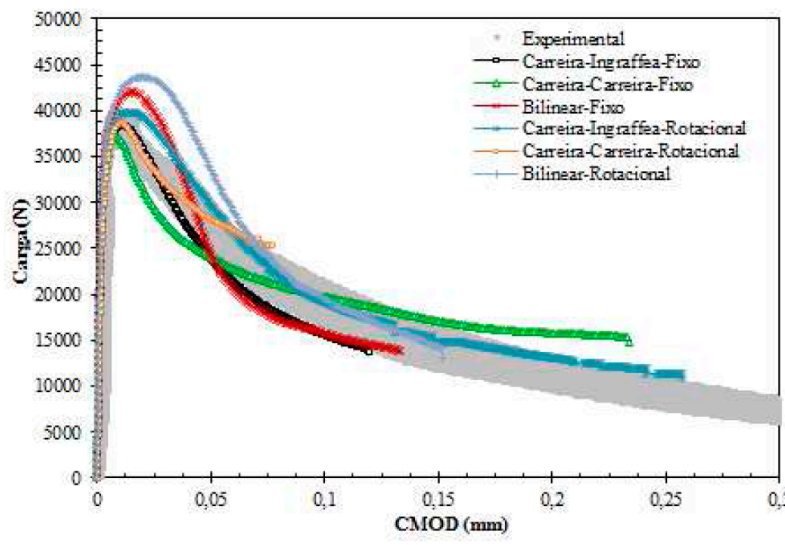

(a) Valores de CMOD

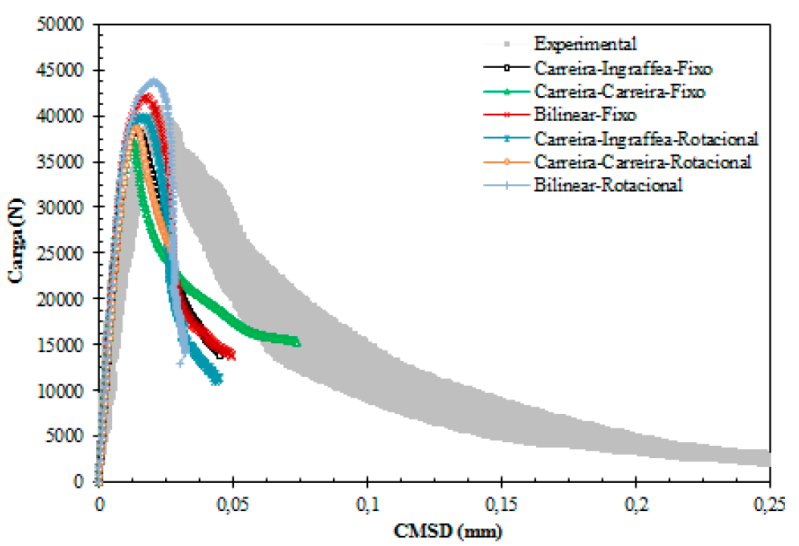

(b) Valores de CMSD

O melhor desempenho dos Modelos CarreiraIngraffea, fixo e rotacional, na descrição do regime pós-fissuração, em modo de abertura, pode ser explicado pela relação direta que o ramo descendente da curva tensão-deformação deste modelo tem com a energia de fratura do material, enquanto que no Modelo Carreira-Carreira o referido ramo descendente depende da diferença entre $\varepsilon_{c}$ e $f_{t} / E_{0}$, sem qualquer relação com a energia de fratura do material. O desempenho superior dos modelos Carreira-Ingraffea permite concluir que, para descrição do regime de abrandamento, a lei exponencial proposta por BOONE e INGRAFFEA (1987) é mais adequada que a polinomial, proposta por CARREIRA e CHU (1986). 
O desempenho inferior dos modelos na descrição do regime pós-fissuração, em modo de deslizamento no plano, pode ser atribuído à ausência de qualquer mecanismo explícito para estimar a rigidez transversal neste regime. Em todos os modelos, a estimativa da rigidez tranversal é feita por combinação implícita dos módulos de elasticidade longitudinais no sistema local.

O pior desempenho dos Modelos Bilineares, tanto na estimativa da carga limite quanto na descrição do abrandamento, se explica pela avaliação do decaimento da rigidez incremental pós-fissuração. Enquanto nos demais modelos, este decaimento é mais acentuado logo após a fissuração, suavizandose ao longo do processo, no Modelo Bilinear, tal decaimento é constante. Provavelmente, o uso de uma lei trilinear seria mais adequada.

A superestimativa do limite de resistência e da ductilidade, obtida com os modelos de direção rotacional, pode ser explicada pela maior capacidade de deformação que estes modelos têm em relação aos modelos fixos, uma vez que a direção de fissuração é livremente alterada durante todo o processo de propagação. Isto indica que a hipótese de que a direção da fissura permanece inalterada após o início da propagação é mais adequada.

\section{Considerações Finais}

Vários modelos para representar o concreto foram aqui apresentados, resumindo-se importantes trabalhos sobre o tema. Inúmeros estudos, sejam teóricos, experimentais e/ou numéricocomputacionais, já foram desenvolvidos sob diversas perspectivas, por meio da formulação de novos modelos ou do aprimoramento de modelos existentes. O emprego do MEF é fundamental para estudar o complexo comportamento do concreto, pois modelos analíticos são de difícil obtenção e limitados a casos simplificados. Dentre os modelos para análise pelo $\mathrm{MEF}$, aqueles que consideram o meio degradado como contínuo vêm sendo desenvolvidos desde a década de 60 e, hoje, são convenientemente segmentados sob as denominações: Modelos de Fissuração Distribuída; Modelos de Dano e Modelos de Microplanos. Analisando os estudos mais atuais, verifica-se a tendência de eliminar esta segmentação, unificandose os diversos modelos em uma estrutura teórica comum capaz de tratar de forma genérica a degradação do meio, conforme a recente proposta de PENNA (2011).

Um esquema para formulação de Modelos de Fissuração Distribuída ortotrópicos foi aqui apresentado e três modelos, concebidos segundo esse esquema, foram detalhados. Os três modelos foram então usados para modelar uma viga e os resultados demonstraram excelente correspondência com os obtidos experimentalmente.

É importante destacar, entretanto, que nenhum estudo foi feito para verificar uma limitação comum dos modelos do MEF para concreto: a dependência da malha de elementos finitos. Com o refinamento da malha, a resposta caracteriza a estrutura em análise como menos resistente e mais frágil. Vários estudos têm atribuído tal dependência à indução numérica de localização de deformações: se a deformação se localiza em um elemento, por exemplo, a resposta do modelo passa a depender do tamanho do mesmo.

Estes fenômenos de localização de deformações, recorrentes na modelagem do concreto, têm sido tratados com o uso de modelos não-locais (BAŽANT e PIJAUDIER-CABOT, 1988). Outras possibilidades para tentar sanar esta dificuldade têm surgido recentemente, podendo-se citar: o uso de elementos finitos com descontinuidade forte embutida (MANZOLI e SHING, 2006); o uso de métodos sem malha (BARROS, 2002) e a formulação de modelos constitutivos utilizando-se teorias de contínuos generalizados (FUINA, 2009).

\section{Agradecimentos}

Os autores agradecem o apoio financeiro em forma de fomento à pesquisa concedido pela 
FAPEMIG (Fundação de Amparo à Pesquisa do Estado de Minas Gerais) e pela CAPES (Coordenação de Aperfeiçoamento de Pessoal de Nível Superior).

\section{Referências}

ALVES, M., YU, J., JONES, N., 2000. On the elastic modulus degradation in continuum damage mechanics. Computers \& Structures 76, 703-712.

BARAIN, J. M., MARI, A., 2007. Multiaxial-coupled analysis of rc cross-sections subjected to combined forces. Engineering Structures 29, 1722-1738.

BARROS, F. B., 2002. Métodos sem malha e método dos elementos finitos generalizados em análise não-linear de estruturas. Tese de Doutorado, Universidade de São Paulo, São Carlos.

BAZANT, Z. P., 1976. Instability, ductility and size effect in strain-softening concrete. Journal of the engineering mechanics division 102, 331-344.

BAZANT, Z. P., CEDOLIN, L., 1979. Blunt crack band propagation in finite element analysis. Journal of the Engineering Mechanics Division 105, 297-315.

BAZANT, Z. P., CEDOLIN, L., 1980. Fracture mechanics of reinforced concrete. Journal of the Engineering Mechanics Division 106, 1287-1306.

BAZANT, Z. P., CEDOLIN, L., 1983. Finite element modeling of crack band propagation. Journal of Structural Engineering 109 (1), 69-92.

BAZANT, Z. P., GAMBAROVA, P. G., 1984. Crack shear in concrete: crack band microplane model. Journal of Structural Engineering 110 (9), 2015-2035.

BAZANT, Z. P., OH, B. H., 1983. Crack band for fracture of concrete. Materiaux ET Constructions 16 (93), 155177.

BAZANT, Z. P., OH, B. H., 1985. Microplane model for progressive fracture of concrete and rock. Journal of Engineering Mechanics 111 (4), 559-582.

BAZANT, Z. P., PIJAUDIER-CABOT, G., 1987. Softening in reinforced concrete beams and frames. Journal of Structural Engineering, ASCE 113 (12), 23332347.

BAZANT, Z. P., PIJAUDIER-CABOT, G., 1988. Nonlocal continuum damage, localization instability and convergence. Journal of Applied Mechanics 55 (2), 287293.
BELNOUE, J. P., NGUYEN, G. D., KORSUNSKY, A. M., 2007. A one-dimensional nonlocal damageplasticity model for ductile materials. Letters in Fracture Micromechanics 144, 53-60.

BLAAUWENDRAAD, J., 1972. Realistic analysis of reinforced concrete framed structures. HERON 18 (4), $1-23$.

BOONE, T., INGRAFFEA, A. R., 1987. Simulation of the fracture process at rock interfaces. In: Proceedings of the fourth international conference in Numerical Methods in fracture Mechanics. pp. 519-531.

BOONE, T., WAWRZYNEK, P. A., INGRAFFEA, A. R., 1986. Simulation of the fracture process in rock with application to hydrofracturing. International Journal of Rock Mechanics and Minig Science 23 (3), 255-265.

BORGES, J. U. A., BITTENCOURT, T. N., 2005. Efeito de escala na ductilidade do concreto sob compressão uniaxial e vigas armadas sob flexão. Revista IBRACON de Estruturas 1 (2), 187-213.

BREKELMANS, W. A. M., SCHEURS, P. J. G., DE VREE, J. H. P., 1992. Continuum damage mechanics for softening of brittle materials. Acta Mechanica 93, 133143.

CAMARA, L., FERREIRA, R. L., MOREIRA, R. N., PENNA, S. S., SALIBA, S. S. e FONSECA, M. T. INSANE - Versão 2.0. XXVII CILAMCE, UFAL, Maceió 2008; p.1-20.

CAROL, I., RIZZI, E., WILLAM, K., 1994. A unified theory of elastic degradation and damage based on a loading surface. International Journal of Solids and Structures 31 (20), 2835-2865.

CAROL, I., 1999. New developments in elastic degradation and damage: anisotropic formulations and evolution laws in pseudo-log space. In: MECOM 99. Mendoza, Argentina, pp. 3-16.

CAROL, I., JIRASEK, M., BAZANT, Z., 2001a. A thermodinamically consistent approach to microplane theory. Part I. Free energy and consistent microplane stresses. International Journal of Solids and Structures 38, 2921-2931.

CAROL, I., PRAT, C., BAZANT, Z. P., 1992. New explicit microplane model for concrete: Theoretical aspects and numerical implementation. International Journal of Solids Structures 29 (9), 1173-1191.

CAROL, I., RIZZI, E., WILLAM, K., 2001a. On the formulation of anisotropic elastic degradation. I. Theory based on a pseudo-logarithmic damage tensor rate. International Journal of Solids and Structures 38, 491518. 
CAROL, I., RIZZI, E., WILLAM, K., 2001b. On the formulation of anisotropic elastic degradation. II. Generalized pseudo-rankine model for tensile damage. International Journal of Solids and Structures 38, 519546.

CARREIRA, D. J., CHU, K.-H., 1985. Stress-strain relationship for plain concrete in compression. American Concrete Institute Journal 82 (6), 797-804.

CARREIRA, D. J., CHU, K.-H., 1986. Stress-strain relationship for reinforced concrete in tension. American Concrete Institute Journal 83 (1), 21-28.

CAUVIN, A., TESTA, R. B., 1999. Damage mechanics: basic variables in continuum theories. International Journal of Solids and Structures 36, 747-761.

CHEN, W. F., 1982. Plasticity in Reinforced Concrete. McGraw-Hill Book Company, Nova York, EUA.

CHOW, C. L., WANG, J., 1987. An anisotropic theory of continuum damage mechanics for ductile fracture. Engineering Fracture Mechanics 27 (5), 547-558.

COMI, C., 2001. A non-local model tension and compression damage mechanims. European Journal of Mechanics - A/Solids 20, 1-22.

DARWIN, D., PECKNOLD, D. A., 1976. Analysis of rc shear panels under cyclic loading. In: Journal of the structural division, ASCE. pp. 355-369.

DE BORST, R., 2002. Fracture in quasi-brittle materials: a review of continuum damage-based approaches. Engineering Fracture Mechanics 69, 95-112.

DE BORST, R., GUTIÉRREZ, M. A., 1999. A unified framework for concrete damage and fracture models including size effects. International Journal of Fracture 95, 261-277.

DE BORST, R., NAUTA, P., 1985. Non-orthogonal cracks in a smeared finite element model. Engineering Computations 2, 35-46.

DE VREE, J. H., BREKELMANS, W. A. M., VAN GILS, M. A. J., 1995. Comparison of nonlocal approaches in continuum damage mechanics. Computers \& Structures 55 (4), $581-588$.

DESMORAT, R., GATUINGT, F., RAGUENEAU, F., 2007a. Nonlocal anisotropic damage model and related computational aspects for quasi-brittle materials. Engineering Fracture Mechanics 74, 1539-1560.

DUNDAR, C., TOKGOZ, S., TANRIKULU, A. K., BARAN, T., 2008. Behaviour of reinforced and concreteencased composite columns subjected to biaxial bending and axial load. Building and Environment 43, 1109-1120.
FALEIRO, J., OLLER, S., BARBAT, A., 2008. Plasticdamage seismic model for reinforced concrete frames. Computers \& Structures 86, 581-597.

FUINA, J. S., Abril 2009. Formulações de modelos constitutivos de microplanos para contínuos generalizados. Tese de Doutorado, Universidade Federal de Minas Gerais, Belo Horizonte, MG, Brasil.

GHRIB, F., TINAWI, R., 1995. Nonlinear behavior of concrete dams using damage mechanics. Journal of Engineering Mechanics 121 (4), 513-527.

HE, W., WU, Y.-F., LIEW, K. M., 2008. A fracture energy based constitutive model for the analysis of reinforced concrete structures under cyclic load. Computational Methods in Applied Mechanics Engineering 197 (51-52), 4745-4762.

HILlERBORG, A., MODÉER, M., PETERSON, P.E., 1976. Analysis of crack formation and crack growth in concrete by means of fracture mechanics and finite elements. Cement and Concrete Research 6, 773-782.

JIRÁSEK, M., PATZ_AK, B., 2002. Consistent tangent stiffness for nonlocal damage models. Computers \& Structures 80, 1279-1293.

JIRÁSEK, M., ZIMMERMANN, T., 1998. Rotating crack model with transition to scalar damage. Journal of Engineering Mechanics 124, 277-284.

JUNIOR, F. S., VENTURINI, W. S., 2007. Damage modelling of reinforced concrete beams. Advances in Engineering Software 38, 538-546.

KUHL, E., STEINMANN, P., CAROL, I., 2001. A thermodinamically consistent approach to microplane theory. Part II. Dissipation and inelastic constitutive modeling. International Journal of Solids and Structures 38, 2933-2952.

LEE, J. S., CHOI, I.-Y., CHO, H.-N., 2004. Modeling and detection of damage using smeared crack model. Engineering Structures 26, 267-278.

LEE, U., LESIEUTRE, G. A., FANG, L., 1997. Anisotropic damage mechanics based on strain energy equivalence and equivalent elliptical microcracks. International Journal of Solids Structures 34, 4377-4397.

LEMAITRE, J., 1992. A Course on Damage Mechanics. Springer, Berlim.

LEMAITRE, J., DESMORAT, R., 2005. Engineering Damage Mechanics - Ductile, Creep, Fatigue and Brittle Failures. Springer, Berlim.

LEMAITRE, J., DUFAILly, J., 1987. Damage measurements. Engineering Fracture Mechanics 28 (5/6), 643-661. 
LEUKART, M., RAMM, E., 2002. An alternative split within the microplane material model. In: H.A. Mang, F. R. e. J. E. (Ed.), Fifth World Congress on Computational Mechanics. Viena, Austria.

LEUKART, M., RAMM, E., 2006. Identification and interpretation of microplane material laws. Journal of Engineering Mechanics 132 (3), 295-305.

LI, Y.-J., ZIMMERMANN, T., 1998. Numerical evaluation of the rotating crack model. Computers \& Structures 69, 487-497.

MANZOLI, O. L., OLIVER, J., DIAZ, G., HUESPE, A. E., 2008. Análise tridimensional de elementos estruturais de concreto armado via elementos finitos com descontinuidades incorporadas. Revista IBRACON de Estruturas e Materiais 1 (1), 58-83.

MANZOLI, O. e SHING, P. A general technique to embed non-uniform discontinuities into standard solid finite elements. Computers \& Structures, v. 84, 2006; p.742-757.

MATA, P., OLLER, S., BARBAT, A. H., 2007. Static analysis of beam structures under nonlinear geometric and constitutive behavior. Computer methods in applied mechanics and engineering 196, 4458-4478.

MAZARS, J., 1984. Application de la mécanique de l'endommagement au comportement non lineaire et á la rupture du béton de structure. Tese de Doutorado, Université Paris, Paris, citado por Lemaitre (1992).

MENIN, R. C., TRAUTWEIN, L. M., BITTENCOURT, T. N., 2009. Modelos de fissuração distribuída em vigas de concreto armado pelo método dos elementos finitos. Revista IBRACON de Estruturas e Materiais 2 (2), 166200.

MONNIER, T., 1970. The moment-curvature relation of reinforced concrete. HERON 18 (2), 1-101.

MOSALAM, K. M., PAULINO, G. H., 1997. Evolutionary characteristic length method for smeared cracking finite element models. Finite Elements in Analysis and Design 27, 99-108.

NGO, D., SCORDELIS, A. C., 1967. Finite element analysis of reinforced concrete beams. American Concrete Institute Journal 67, 152-163.

NILSON, A. H., 1968. Nonlinear analysis of concrete by the finite element method. Journal of American Concrete Institute 65, 757-766.

OZBOLT, J., LI, Y., KOZAR, I., 2001. Microplane model for concrete with relaxed kinematic constraint. International Journal of Solids and Structures 38, 26832711.
PALIGA, C. M., FILHO, A. C., REAL, M. V., 2007. Modelo de elementos finitos para análise numérica de estruturas de concreto armado reforçadas. Revista IBRACON de Estruturas 3 (2), 177-200.

PAVAN, R. C., CREUS, G. J., MAGHOUS, S., 2009. A simplified approach to continuous damage of composite materials and micromechanical analysis. Composite Structures 91, 84-94.

PENNA, S. S., Novembro 2011. Formulação multipotencial para modelos de degradação elástica: unificação teórica, proposta de novo modelo implementação computacional e modelagem de estruturas de concreto. Tese de Doutorado, Universidade Federal de Minas Gerais, Belo Horizonte, MG, Brasil.

PETRANGELI, M., OZBOLT, J., 1996. Smeared crack approaches - material modeling. Journal of Engineering Mechanics 122 (6), 545-554.

PI, Y.-L., BRADFORD, M., TIN-LOI, F., GILBERT, R., 2007. Geometric and material nonlinear analysis of elastically restrained arches. Engineering Structures 29, 283-295.

PITANGUEIRA, R. L. S., Agosto 1998. Mecânica de estruturas de concreto com inclusão de efeitos de tamanho e heterogeneidade. Tese de Doutorado, Pontifícia Universidade Católica do Rio de Janeiro, Rio de Janeiro.

PITANGUEIRA, R. L. S., FONSECA, F. T., FUINA, J. S., CAMARA, L., FERREIRA, R. L., MOREIRA, R. N., PENNA, S. S., SALIBA, S. S. e FONSECA, M. T. INSANE - Versão 2.0. XXVII CILAMCE, UFAL, Maceió 2008; p.1-20.

PITUBA, J. J. C., 1998. Estudo e aplicação de modelos constitutivos para o concreto fundamentados na mecânica do dano contínuo. Dissertação de Mestrado, Universidade de São Paulo, São Carlos.

PITUBA, J. J. C., 2003. Sobre a formulação de um modelo de dano para o concreto. Tese de Doutorado, Universidade de São Paulo, São Carlos.

PROENÇA, S. P. B., 1988. Sobre modelos matemáticos do comportamento não-linear do concreto: Análise crítica e contribuições. Tese de Doutorado, Universidade de São Paulo, São Carlos.

PROENÇA, S. P. B., PITUBA, J. J. C., 2008. Sobre a formulação de um modelo de dano para o concreto. Cadernos de engenharia de estruturas 10 (47), 117-1146.

RASHID, Y. R., 1968. Ultimate strenght analysis of prestressed concrete pressure vessels. Nuclear Engineering and Design 7, 334-344. 
RIZZI, E., CAROL, I., WILLAM, K., 1995. Localization analysis of elastic degradation with application to scalar damage. Journal of Engineering Mechanics 121 (4), 541554.

ROTS, J. G., 1988. Computational modeling of concrete fracture. Tese de Doutorado, Delft University of Technology, Delft, Holanda.

ROTS, J. G., DE BORST, R., 1987. Analysis of mixedmode fracture in concrete. Journal of Engineering Mechanics 113 (11), 1739-1758.

ROTS, J. G., NAUTA, P., KUSTERS, G. M., BLAAUWENDRRA, J., 1985. Smeared crack approach and fracture localization in concrete. HERON 30 (1), $1-48$.

SCOTTA, R., VITALIANI, R., SAETTA, A., ONATE, E., HANGANU, A., 2002. A scalar damage model with a shear retention factor for the analysis of reinforced concrete structures: theory and validation. Computers \& Structures 79, 737-755.

SCHLANGEN, E. Experimental and Numerical Analysis of Fracture Processes in Concrete, 1993, Tese de Doutorado, Delft University of Technology.

SHAYANFAR, M. A., KHEYRODDIN, A., MIRZA, M. S., 1997. Element size effect in nonlinear analysis of reinforced concrete members. Computers \& Structures 62 (2), 339-352.

SILVA, P. P. R., Fevereiro 2002. Implementação de modelos de microplanos para análise não-linear de estruturas de concreto, uma abordagem orientada a objetos. Dissertação de Mestrado, Universidade Federal de Minas Gerais, Belo Horizonte, MG, Brasil.

SIMÃO, W. I. S., Agosto 2003. Modelos de armadura e aderência para análise não linear de concreto armado. Dissertação de Mestrado, Universidade Federal de Minas Gerais, Belo Horizonte, MG, Brasil.
SRIKANTH, M., KUMAR, G. R., GIRI, S., 2007. Moment curvature of reinforced concrete beams using various confinement models and experimental validation. Asian Journal of Civil Engineering (Building and Housing) 8 (3), 247-265.

SUIDAN, M., SCHNOBRICH, W. C., 1973. Finite element analysis of reinforced concrete. In: Journal of the structural division, ASCE. pp. 2109-2121.

SWARTZ, S. E., HU, K.-K., JONES, G. L., 1978. Compliance monitoring of crack growth in concrete. In: Journal of the engineering mechanics division, ASCE. pp. 789-800.

TAYLOR, G. I., 1938. Plastic strains in metals. Journal of the Institute of Metals 62, 307-324. Citado por Bazant e Planas (1998).

VOYIADJIS, G. Z., TAQIEDDIN, Z. N., KATTAN, P. I., 2008. Anisotropic damage-plasticity model for concrete. International Journal of Plasticity 24, 1946-1965.

WEIHE, S., KRÖPLIN, B., DE BORST, R., 1998. Classification of smeared cracking models based on material and structural properties. International Journal of Solid and Structures 35 (12), 1289-1308.

XIANG, Z., SWOBODA, G., CEN, Z., 2002. Identification of damage parameters for jointed rock. Computers \& Structures 80, 1429-1440.

YANG, Y. B., SHIEH, M. S., 1990. Solution method for nonlinear problems with multiple critical points. AIAA Journal 28 (12), 2110-2116. 
